\title{
Subjective and Behavioral Presence Measurement and Interactivity in the Collaborative Augmented Reality Game TimeWarp
}

\author{
Jennifer Klatt ${ }^{*}$, Astrid M. von der Pütten ${ }^{1}$, Simon Ten Broeke ${ }^{1}$, Roderick McCall , Nicole C. Krämer ${ }^{1}$, Richard \\ Wetzel $^{3}$, Lisa Blum ${ }^{3}$, Leif Oppermann ${ }^{3}$, Johannes Klatt ${ }^{1}$ \\ ${ }^{1}$ University of Duisburg-Essen, Forsthausweg 2, 47057 Duisburg, Germany, \\ 2 SnT, University of Luxembourg, 6 rue Richard Coundenhove-Kalergi, L-1359, Luxembourg-Kirchberg \\ ${ }^{3}$ Fraunhofer FIT, Schloss Birlinghoven, 53754 Sankt Augustin, Germany \\ \{jennifer.klatt; astrid.von-der-puetten; nicole.kraemer; johannes.klatt\}@uni-due.de, simon.ten-broeke@stud.uni-due.de, \\ roderick.mccall@uni.lu, \{richard.wetzel; lisa.blum; leif.oppermann\}@fit.fraunhofer.de
}

\section{Corresponding author:}

Astrid von der Pütten, astrid.von-der-puetten@uni-due.de

Telephone: +49203379 - 1330

Telefax: +49203379- 3670 
Highlights:

- pointing and verbal responses to virtual content are correlated negatively to presence

- $\quad$ perceived interaction possibilities predict perceived social presence of virtual characters

- gamers' relationship status does not influence presence 


\title{
Subjective and Behavioral Presence Measurement and Interactivity in the Collaborative Augmented Reality Game TimeWarp
}

\begin{abstract}
Presence measurements are traditionally using a variety of subjective and objective measures. However, constraints often result in subjective measurements using questionnaires as a key method of data collection. In this paper we present a study of 44 participants of a collaborative augmented reality game known as TimeWarp which used both subjective and objective behavioral measures where both video recordings and self-reports about feelings of presence were compared. We further investigated the influence of the collaborative nature of the game and of subjectively perceived interactivity on perceived presence. Our findings indicate that pointing behavior and verbal responses to virtual content within an augmented reality scene are correlated negatively to sense of presence. Our results suggest that certain behavioral measures correlate with subjective feelings of presence and can predict the latter in the augmented reality game. With regard to collaborative game play and interactivity on presence we found no influence for collaborative gameplay, however, the interaction possibilities perceived by the participants predicted their experience of social presence with the virtual characters in the game. Implications for presence research are discussed.
\end{abstract}

Keywords: Presence in Games, Behavioral Measures, Augmented Reality, Collaborative Games, Interactivity 


\section{Introduction}

Mobile location-aware augmented reality (AR) games present a number of challenges for researchers in the field of presence. For example laboratory based studies are often impossible due to the close coupling of the game with the real environment, therefore tests with systematically controlled variables and removing possible external influences remains problematic. Furthermore, the notion of presence within such games still requires considerable work, as by default people are "present" in reality but unlike virtual environments are not seeking to remove themselves from reality. As a result the sense of presence in any AR game is a mix of both the real and virtual space.

The TimeWarp game was developed as part of the EC funded IPCity project by Fraunhofer FIT (see [1] for a previous version of the game). The game itself takes place in the City of Cologne and utilizes the history and locations of the city along with folklore. As such it is an example of the challenges faced by researchers and also provides a usecase which explores how specific design decisions within augmented reality games impact upon user behavior and their sense of presence. The game uses a compelling narrative which brings together rich 3D augmented reality content with characters to create an engaging storyline.

The goal of the presented study is threefold. First the study was conducted to examine if and which behavioral elements correlate with the player's sensation of presence. Therefore, the results of an analysis of the players' behavior based on video recordings and their self-reported experience of presence based on presence questionnaires were compared. Second, the study examines the influences of the perceived interaction possibilities with virtual characters on perceived social presence of these characters. And third, we explore the role of socio-demographic variables and of the relationship status of the collaborative players on their experienced sense of presence on different presence dimensions.

With regard to applied research, it aims at analyzing the forms of presence that can be found in augmented reality games. Concerning more fundamental research on presence, it tries to provide evidence which objectively measurable aspects can be seen as a means to reliably assess presence via other aspects than self-report.

\section{Theoretical Background}

In order to address the key issues of measuring presence in mobile location-aware augmented reality games the following section contains three parts: Presence as a psychological concept, typical measurements of presence, and an overview of augmented reality and collaborative game play in relation to presence.

\subsection{Presence}

The concept of presence is defined in different ways: First introduced by Lombard and Ditton in 1997, the term presence was used for a "perceptual illusion of nonmediation" [2, p.3]. This means that the recipient who is subject to the feeling of presence is not aware on the medium which is being used i.e. they are not aware of the technology upon 
which they are experiencing their new reality. Witmer and Singer [3] describe presence differently and define presence as a phenomenon where percipients have the experience of being in another place mentally, although the actual body does not move. Therefore, presence is also known as the sensation of "being there" [3; 4]. Moreover, the perceived objects are part of presence definitions [6, p.37]: "a psychological state in which virtual (para-authentic or artificial) objects are experienced as actual objects in either sensory or nonsensory ways".

Two factors that have a high impact on sense of presence are the level of interactivity and realism. The first one of course is especially interesting for presence in games, since interactivity is a crucial criterion for video games [7]. It is the main characteristic that distinguishes games from other media. Several studies examined the relation of interactivity and presence and found that playing a game leads to a higher sense of presence than observing a game [8; 9]. This might be related to the fact that interactivity also leads both to a higher degree of entertainment [10] and arousal [11]. Therefore, interactivity can be seen as an influencing component for the sensation of presence.

The second aspect is realism. A higher degree of realism that increases perceptions with presence can be reached in several ways like, for instance, visual realism [12], $1^{\text {st }}$ person view compared to $3^{\text {rd }}$ person view [13; 14], the usage of sound [15], the virtual representation of the body [16;17] or behavioral realism which is defined as "the degree to which virtual humans and other objects within [Immersive Virtual Environments] behave as they would in the physical world" [18, p.112]. Therefore, an augmented reality game can create a sense of presence because of both the interactivity and the realism that it contains due to a) the mixture of real-world elements and the behavioral realism of virtual objects and $\mathrm{b}$ ) the $1^{\text {st }}$ person view on the real and virtual objects.

The concept of presence usually contains several very different facets of one phenomenon. Therefore, several subconcepts have been developed that try to split the presence concept into clear distinguished aspects. Very common are the two sub-concepts: physical presence and social presence. Physical presence can be described as ,the sense of being there" [19, p. 150] and social presence as ,the sense of being together with another" [19, p. 151] or as "the illusion of shared physical space" [20, p. 281]. Since TimeWarp is an augmented reality game the aspect of physical presence and the question whether people on the one hand experience the feeling of being in the game and on the other hand perceive the augmented objects and characters as real physical objects and characters is of great importance. Moreover, in TimeWarp people interact with a human partner and also with augmented characters, hence social presence is also relevant to our investigation.

In the context of the TimeWarp game another facet that has not yet been defined in a sub-concept of presence is temporal presence. It is especially interesting for the TimeWarp game since in TimeWarp players are time travelers and switch from one time period to another throughout the game. Temporal presence describes the sensation of being in the time of the perceived content. For this study measures were developed for temporal presence since it is not addressed in other literature yet. In the context of the TimeWarp game, the occurrence of temporal presence is presumably also an indicator for presence in general. 
All of these concepts have general presence aspects in common but differ in their focus on a specific presence aspect.

\subsection{Measuring Presence}

Within the presence community it is acknowledged that the measurement of presence faces various challenges in developing valid and reliable measures of presence. Presence measures can differ in several ways, e.g. if it is measured during the presence experience or afterwards. Current research found that this leads to no effects on the results [21]. However, mostly relevant for this paper is the differentiation between subjective measures and objective measures [22].

Subjective measures offer various benefits. They are easy and inexpensive to use. They also seem to be valid measures as they were designed to measure presence in the way the user perceived and feels presence. Since presence is defined as a psychological construct thus self-report measures seem to be a reasonable approach. However, there are several limitations to these subjective measures. Firstly, they are prone to the well-known demand characteristics. Participants try to guess what the researchers are examining and which outcome the investigator expects and then answer according to or contrary to these predictions. In addition, reliability problems have been shown [23]. Second, presence is regarded as a phenomenon which occurs during the reception of or the interaction with media, but selfreported measures are mostly post-experimental assessments of this experience. However, there are techniques that use a real-time approach to measure presence [e.g. 23], but these procedures are suspected to interfere with the presence experience itself. Third, researchers use a broad range of different scales which hinders comparison across studies.

In contrast, objective measures can be assessed during the experience of presence itself because they are based on participants' physiological or behavioral responses (e.g. skin conductance, heart rate, respiration, posture, proxemics, gestures). One way to use objective measures is to relate the behavior or physiological responses shown towards a real object with those shown towards a virtual object. For instance, Bailenson showed that people apply proxemics rules to a virtual human and keep an interpersonal distance to the virtual character comparable to a real human being [24]. In this case, participants are only presented virtual stimuli and their responses are then compared to responses usually shown towards other humans. Another way of relational measure is the simultaneous presentation of real and virtual stimuli as suggested by Prothero, Parker, Furness, and Wells [25]. "The degree to which subjects respond to the virtual cues rather than the real ones indicates presence perceptions." [26, p.6]. However, also objective measures have disadvantages. They are often expensive in terms of technical equipment which is needed to conduct these assessments and also in terms of time needed for analysis. Additionally, as a precondition for these forms of measurements the relation between the behavior and the concept of presence needs to be clear. A way to resolve this problem is to establish the relation between both approaches clearly. Studies can then validate self-reported measures by additionally using behavioral measures and vice versa. 
Behavioral measures that were not related to psycho physiological feedback were used rather rarely so far, so some examples should be presented in detail: Slater, Usoh, and Chrysanthou [27] showed a relation between the pointing behavior of a person in a virtual reality and perceived presence. They asked participants to either point to a virtual object or a real one without letting the participant know which one they meant. If they chose the virtual object it was interpreted as a higher degree of presence in the virtual environment. Another study by Freeman, Avons, Meddis, Pearson, and IJsselsteijn [28] chose the body posture as an operationalization of presence when the participants watched the record of a car race. Especially the operationalization of presence in the latter study seems rather obvious. Slater et al. [29] claim that behavioral measures should be used only when there is an obvious behavior that can be shown when perceiving the stimulus.

In this paper this approach will be applied. Behavioral measures will be compared to subjective presence ratings by the participants. By this, the potential of the behavior to serve as an indicator of perceived presence will be examined.

\subsection{Augmented Reality}

Augmented reality (AR) is a form of a mix between real world and virtual reality (VR). A virtual reality lets a user interact with a virtual environment. „Whereas VR is the construction of a synthetic environment, AR extracts information from the real world and augments it" [30, p.19]. AR application mix real environments with virtual objects like visual, acoustic, or haptic elements. "Ideally, it would appear to the user that the virtual and real objects coexisted in the same space" [31, p.336]. This can be done with cameras, monitors, or see-through and head-mounted displays (HMDs) [32]. According to Höhl [33, p. 12], monitor-based systems are „less suitable for immersive display than HMDs“. Additionally to the display, tracking systems e.g. for head movements or other input devices can be used to make the experience more realistic. Therefore, AR has a possibility to create an environment with which the user can interact in a more natural way [34].

AR-applications can be found in several domains: The car industry for example develops prototypes often using AR environments [33]. Research on how to improve common techniques with using AR is conducted in the area of medicine, e.g. for biopsies [35]. Another area where AR is used is gaming. Several research prototypes, such as the early ARQuake [36], explored the space of possibilities. The game industry recently started to develop constrained augmented reality games like Nintendo for the 3DS [37]. TimeWarp, the game used in this study, is an example of a mobile outdoor mixed reality game developed for pervasive computing and presence research. It is introduced in more detail in the method section.

\subsection{Collaborative Games and Presence}

Games that can be or need to be played by more than one player at a time are called collaborative games and require collaborative behavior. Here, the players can not only interact with the virtual world and objects, but also with their interaction partners. „Collaborative activities require co-actors to articulate - distribute responsibilities, explain, 
guide, align, clarify misunderstandings [...]“ [38]. The collaboration can take place in informal encounters as well as in more organized regularly cooperating groups [39]. Especially in massive multiplayer games, the number of the interacting players influences the interaction type. Nardi and Harris [39] report that the variety of different collaborations increases enjoyment of the game as well as provides rich learning opportunities. Another important aspect for the interaction between the players is the real-life relationship state. For example, they can be either friends or strangers. It was found that compared to playing video games with a stranger, playing with a friend led to greater presence and arousal [40].

Collaboration in augmented reality games has some special characteristics and challenges. "The collaborative AR allows multiple participants to share a physical space surrounding them and a virtual space, visually registered with the physical one" [41]. With regard to gaming, there are several approaches which use AR settings, e.g. the $\mathrm{AR}^{2} \mathrm{Hockey}$ system [41] allows two players to share the experience of an augmented hockey game and play together as opponents. Similarly, one player fights against virtual opponents placed within the game by the second player in the AR-game Multiple-Invading Nefarious Demons (MIND; [42]) It is noteworthy that -in contrast to the presented games- in the TimeWarp game used for this study two players also play together, but share a mutual goal that can only be achieved with cooperation. The players move as a team through the real world and the additionally integrated virtual objects. In contrast to the other games the attention is not directed against objects send by the otherwise "invisible" player, but may be shared between the collaborator and the augmented reality objects.

However, there seems to be a lack on research on the influence of collaboration in games on presence in virtual environments or augmented reality games. Further, former research widely neglected the influence of the players' relationship status on presence in collaborative game play.

\subsection{Research Questions}

As described earlier, the goal of the study was firstly to identify behavioral elements that are linked to the feeling of presence. Therefore, the main research question is:

RQ1: Which behavioral elements accompany the sensation of presence?

Second, we stated that interactivity can be seen as an influencing component for the sensation of presence. In the present study, however, we did not manipulate the interactivity of the game, but aim at examining whether the subjectively perceived possibilities for interaction with virtual objects and characters lead to a higher sense of social presence. Thus, we hypothesize:

H1: Participants who report about a greater feeling of being able to interact with the virtual reality also report a greater sense of social presence (with regard to virtual characters).

And third, regarding the collaborative play, we are interested in whether the perceived social presence of the team player distracts participants from the augmented reality elements resulting in less social presence of virtual characters. 
Moreover, we want to examine the influence on the gamers' relationship status and of socio-demographic variables on perceived presence across various dimensions. We thus pose the following hypothesis and research question:

H2: The concepts social presence of team player and social presence of virtual characters are negatively correlated.

RQ2: Is the experience of presence influenced by the players' relationship status or their gender?

\section{Method}

\subsection{The TimeWarp Game}

The underlying story of the TimeWarp game [1] is time travelling. The players are led by chronoguard Agent Morgan through the game. They are told to collect the robotic Heinzelmaennchen (characters from a Cologne fairy tale, see Figure 1) that are scattered in different time periods which endangers the space time continuum. The universe is on the verge of a collapse if the Heinzelmaennchen are not sent to their native time in the $32^{\text {nd }}$ century. The game is played collaboratively by two players. Each player is equipped with an Ultra-mobile PC (UMPC) to travel through time and interact with the virtual characters and objects. The players share the same goal play in two different modes.

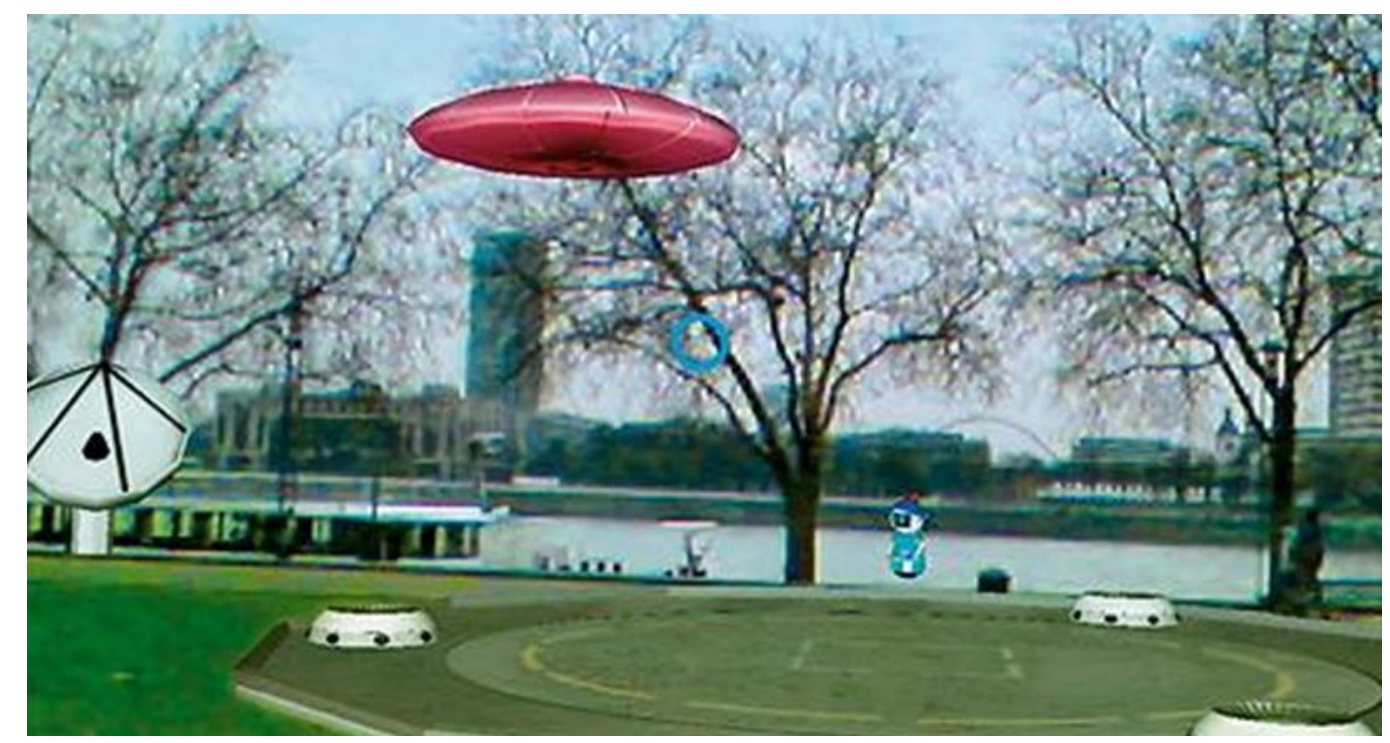

Figure 1 Players view of TimeWarp game with augmented objects/characters.

One device is used by the first player who plays in the augmented reality mode. It is called the "magic lens" that opens the view into different time periods: the screen shows the camera image from the UMPC and virtual elements are rendered on top of it thus creating an AR view of the surroundings. The player's position is tracked with a combination of GPS and inertial and orientation sensors. By aiming the device at virtual elements and pressing a 
button, the player can start interactions like picking something up or talking to a character. The other UMPC is used by the second player as an interactive map showing their current positions and other game elements. Furthermore this player can control all dialogues by selecting from multiple-choice options. The players are free to decide who is playing in which mode and if and when they want to switch. Heinzelmaennchen appear as yellow dots on the map when being approached. Only being in the right time enables visual contact through the "magic lens" device, otherwise the Heinzelmaennchen cannot be seen. In such a case time travelling must be initiated by the second player by placing a virtual timeportal on the map. Then both players have to physically enter the time portal by walking into it. The different time periods (Roman, medieval and future versions of Cologne) can be recognized by time period typical buildings and clothing of the Heinzelmaennchen robots. These mechanic creatures are the main characters of the game. They have seemingly developed self-consciousness which is shown by emotional and moral behavior like bonding and altruism. One task the players have to solve is reuniting two Heinzelmaennchen that want to get married. Opponent to the Heinzelmaennchen is Agent Morgan who recruits the players via a video "livestream" from the $32^{\text {nd }}$ century. Contrary to the computer generated 3D models of the Heinzelmaennchen Agent Morgan is played by an actor. Anthropomorphic characteristics are given to the robot Heinzelmaennchen by using voice artists.

It is important to note that the version of TimeWarp discussed in this paper was tested during 2010 and featured changes from an earlier version [1], namely that certain gaming elements and the devices used for interaction had been changed to reflect an earlier user study. The game had also been extended into a two-player game. Therefore while there are similarities in the underlying mission of the game, its characters and content, the improvements were among other things designed to improve the pace, usability and presence aspects.

A game session lasts for about 60-90 minutes. To test our research, we therefore decided to choose a definite part of the game for the analysis. The chosen part is the wedding of two Heinzelmaennchen robots (see Figure 2). The scene has a non-linear structure as it can be started from the groom's and the bride's location. After a greeting the players are asked to reunite the couple and search for and fetch the groom when meeting the bride first. When the players meet the groom first they are asked to bring him to the bride. Finally Agent Morgan needs to be convinced to wed the couple and the players can attend the ceremony. Afterwards the players are asked to send the Heinzelmaennchen back to Agent Morgan which means that their memory would be erased. The other choice suggested by the Heinzelmaennchen is to send them into a time zone where they can live peacefully together. 


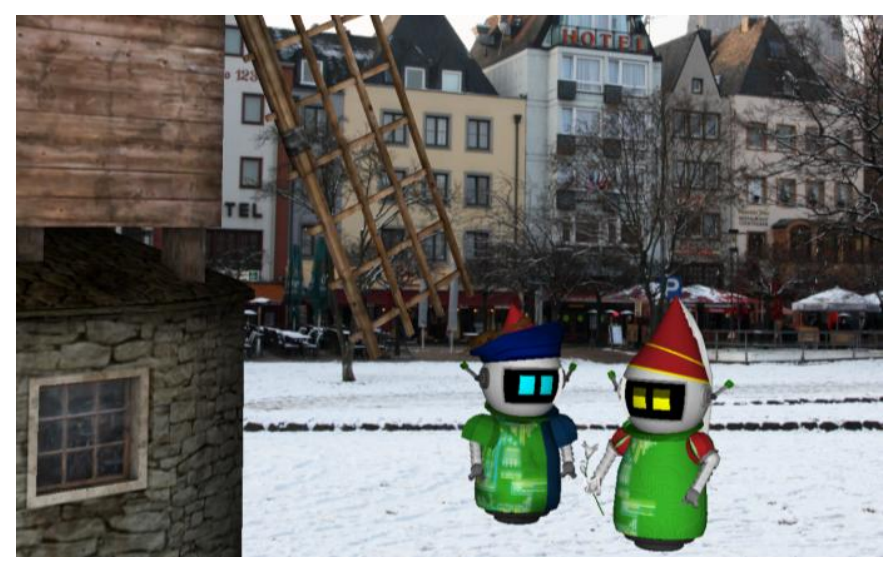

Figure 2 Wedding sequence of TimeWarp game.

\subsection{Participants}

The sample consisted of 66 participants who played the game. Since there are two play modes, only the players who played the augmented reality mode for at least half of the time were chosen $(\mathrm{N}=44,15$ females). The participants played the game in pairs of two players. They played either in same sex pairs (19 participants in male-male and 4 participants in female-female pairs) or mixed sex pairs (21 participants). Also there were different combinations of how well the pairs knew each other: 17 persons played with strangers, 18 persons knew each other or were friends, and 9 participants played together with their partner. All of them had experience with computers and most of them (36 participants) additionally with computer games.

\subsection{Measures}

To measure the experienced presence of the players, a questionnaire was given to the participants. Several aspects of the scales used were inspired and adapted by the MEC Spatial Presence Questionnaire [43] and contained the following aspects of subjective presence ratings:

Social presence of virtual characters: To measure social presence for virtual figures, five items were added that were inspired by the networked minds questionnaire [24] and have been rated on a 7-point Likert scale (Cronbach's alpha $=.778)$. The items were "I felt the presence of another person", "I felt that the person observed me and was aware of my presence", "The person seemed alive to me", "I perceived the person as a computer-generated image, not as a real person", and "The thought that the person was not real came often to my mind".

Social presence of team partner: The social presence of the team partner was measured by two items which were rated on a 7-point Likert scale. The items were "My team partner influenced my behavior", "Throughout the game I was aware of my team partner's presence." 
Willing Suspension of disbelief: From by the MEC questionnaire we added two items of the "willing suspension of disbelief subscale", namely "I didn't really pay attention to the existence of errors or inconsistencies in the game." (reverse), "I concentrated on whether there were any inconsistencies in the game." The items were rated using a 7point Likert scale (Cronbach's alpha $=.803)$.

Involvement: moreover, we included 5 items of the MEC subscale "higher cognitive involvement ("I thought most about things having to do with the game.", "I imagined precisely what it must be like to further explore the time presented in the game.", "I kept wondering whether the presentation in the game could have personal meaning for me.", "I thoroughly considered what the things in the presentation had to do wgith one another.", "The experience activated my thinking.") which were rated also on a 7-point Likert-scale (Cronbach's alpha $=.603$ ).

Immersion: Three items were created that measured the sense of general presence of the game, in particular how players felt as they moved between the game at the start and the end of the experience. They were rated on a 7-point Likert scale. The items were "At the end of the game I felt as if I came back to reality", "At the start of the game I felt as if I were entering a new world", and "While playing the game I felt as if I were part of a game that was not part of the reality".

Virtual interaction: Two items measured the feeling of how much the player was able to interact with virtual content. They were rated on a 7-point Likert scale (Cronbach's alpha $=.774$ ). The items were "I felt as if I could interact with virtual objects" and "I felt as if I could interact with virtual persons".

Originally, the latter was intended to contain not only interaction with virtual objects/persons, but also with real objects, therefore, a third item was created ("I felt as if I could interact with real objects"). However, due to reasons of reliability (Cronbach's alpha with this item $=.616$ ), the third item was excluded. Descriptive statistics tell us, that this is due to a much lower feeling of possible interaction with real objects $(\mathrm{M}=4.07, \mathrm{SD}=1.85)$ than with virtual objects $(\mathrm{M}=4.91, \mathrm{SD}=1.54)$ or persons $(\mathrm{M}=5.20, \mathrm{SD}=1.46)$. With regard to presence this also means that the virtual interaction is more related to the experience of the game.

Temporal presence: Four items were developed to measure temporal presence which means the feeling of how much the player felt present in the time of the game. They were rated on a 7-point Likert scale (Cronbach's alpha = .799). The following items were created: "I visited the future", "I visited the past", "I visited the presence", and "I felt a time shift when moved through time periods".

Behavioral measures: For the behavioral measures video records of the players playing the game were made. The wedding scene was chosen due to the fact that this scene was particularly often referenced as the scene with the most immersive story. The categories that were coded were chosen because they occur in interactions in everyday life and are assumed to occur in interaction with virtual content as well when presence is experienced. Emotional reactions were used as variables as well because research found that they are connected to the experience of presence [4]. In the coding process the number of how often each behavior was shown was specified. Variables that were coded from the recordings include the following verbal and nonverbal reactions shown in relation to virtual content: 
- laughing as a reaction to virtual content,

- verbal answer in a dialogue with virtual content,

- commenting the virtual content,

- smiling about virtual content,

- acoustic sound as a reaction to virtual content,

- turning towards virtual figure, and

- pointing to virtual content.

Noteworthy for the verbal variables is the fact, that the player's were aware that speech was not recognized by the system as all interaction was handled via multiple-choice options. Hence, all of the players' reactions were not necessary for playing the game.

Moreover, we concentrated on verbal and nonverbal behaviors related to the collaborative play with the team partner:

- giving verbal directions,

- "we"-statements,

- "I"-statements,

- $\quad$ look at each other.

\subsection{Procedure}

Participants were introduced to the game and the technology used by a tutorial level moderated by Agent Morgan. Then the game was played for 60-90 minutes and the participants were recorded on video the whole time. Afterwards, a questionnaire followed with questions containing the participant's socio-demographic data, the described measures, and several additional questions. This was followed by an open interview about the experiment that will not be discussed in this paper.

\section{Results}

As a precondition for the other calculations, the group of participants was tested for homogeneity. Since the participants contained persons who played the augmented reality mode from half of the play time till throughout the whole game, it was tested if these groups differ systematically from each other. Four ANOVAS were conducted with the mostly played mode as the independent and the four presence aspects social presence, temporal presence, immersion, and virtual interaction as independent factors. No difference was found, therefore, they can be treated as one group.

In general the game TimeWarp was able to induce a sense of presence during the play. The means of the subjective presence measures hint to neutral or positive presence ratings. Especially two aspects, virtual interaction and the social 
presence of the team partner, show a strong sense of presence (for specific values see Table 1). The means of the behavior shown differ strongly dependent of the participants. As can be seen in the values for standard deviations some participants showed behavior very often whereas most participants almost showed no or very few behavior in interaction with the virtual content. Most commonly were reactions as laughing, smiling, and commenting the virtual content (see values in Table 2).

In sum, the descriptive statistics suggest that this behavior is shown while the players felt the sensation of presence.

Table 1 Means of Presence Ratings

\begin{tabular}{lll}
\hline & $\mathrm{M}$ & $\mathrm{SD}$ \\
\hline Social presence of virtual characters & 3.73 & 1.31 \\
\hline Social presence of team partner & 5.85 & 1.13 \\
\hline Willing suspension of disbelief & 3.99 & 1.42 \\
\hline Higher cognitive involvement & 4.39 & 0.99 \\
Temporal presence & 3.93 & 1.35 \\
Immersion & 4.34 & 1.04 \\
Virtual interaction & 5.05 & 1.35 \\
\hline
\end{tabular}

Table 2 Means of Behavioral Measures

\begin{tabular}{lll}
\hline & $\mathrm{M}$ & $\mathrm{SD}$ \\
\hline Laughing as a reaction to VC & 3.98 & 2.96 \\
Verbal answer in a dialogue with VC & 1.20 & 1.61 \\
Commenting the VC & 7.68 & 4.16 \\
Smiling about VC & 2.98 & 2.68 \\
Acoustic sound as a reaction to VC & 1.55 & 1.53 \\
Turning towards virtual figure & 1.12 & 1.53 \\
Pointing to virtual content & 1.34 & 2.12 \\
Giving verbal directions, & 1.95 & 1.76 \\
"we"-statements, & 4.36 & 3.66 \\
"I"-statements, & 1.95 & 1.76 \\
Look at each other. & 4.25 & 2.21 \\
\hline
\end{tabular}

Abbreviation: $\mathrm{VC}=$ virtual content 


\subsection{Comparing self-report measures \& behavioral measures}

To test the relation between self-reported presence and behavioral elements shown (RQ1) correlations between the aspects social presence of virtual characters, social presence of team partner, temporal presence, immersion, higher cognitive involvement, willing suspense of disbelief, and virtual interaction and the coding from the video records were conducted.

For social presence of virtual characters and for social presence of the team partner no significant correlations occurred. There were also no significant correlations of behavioral measures with immersion, involvement or willing suspense of disbelief.

For temporal presence a correlation was found for the behavior "pointing to something" $(\mathrm{r}=-.388, \mathrm{p}=.009, \mathrm{~N}=44)$. So participants who felt a stronger sense of temporal presence pointed less often to objects and vice versa.

No significant correlations emerged for immersion.

The last presence aspect, virtual interaction, correlated negatively with two behavioral measures: "pointing to virtual content" $(\mathrm{r}=-.392, \mathrm{p}=.009 ; \mathrm{N}=44)$ and "verbal answer in a dialogue with virtual content" $(\mathrm{r}=-.332, \mathrm{p}=.028$, $\mathrm{N}=44$ ). Therefore, participants who felt a stronger sense of the possibility to interact with virtual content gave less verbal answers to the virtual content and pointed less often to objects.

To examine if presence can be predicted by behavior, regression analyses were conducted. The behavioral measures from the video recordings were tested as predictors for the presence aspects social presence of virtual characters, social presence of team partner, higher cognitive involvement, willing suspense of disbelief, temporal presence, immersion, and virtual interaction. None of the behavioral measures could predict social presence of virtual characters or the team partner. Nor were behavioral meausres found to be predictors for involvement or willing suspense of disbelief. For temporal presence "pointing to virtual content" was identified as a predictor (see Table 3 ). Therefore, from the degree of how much participants point to objects it can be predicted how much they feel present at the time of the game.

Table 3 Stepwise regression for Temporal presence

\begin{tabular}{lcccc}
\hline & B & Se B & Beta & Sig \\
\hline Step 1 & & & & \\
Constant & 4.256 & 0.225 & & \\
Pointing to virtual & -0.246 & 0.090 & -.388 & .009 \\
content & & & & \\
\hline Note: $\mathrm{R}=.150$ for step 1, $(\mathrm{ps}>.05)$. & & & \\
\end{tabular}


Immersion could not be predicted by any of the behavioral measures. The best predictor for virtual interaction was "pointing to virtual content" and was in a second step followed by "verbal answer in a dialogue with virtual content" (see Table 4).

Table 4 Stepwise regression for Virtual Interaction

\begin{tabular}{|c|c|c|c|c|}
\hline & B & $\mathrm{Se} \mathrm{B}$ & Beta & Sig \\
\hline \multicolumn{5}{|l|}{ Step 1} \\
\hline Constant & 5.391 & 0.225 & & \\
\hline $\begin{array}{l}\text { Pointing to virtual } \\
\text { content }\end{array}$ & -0.249 & 0.090 & -.392 & .009 \\
\hline \multicolumn{5}{|l|}{ Step 2} \\
\hline Constant & 5.762 & 0.253 & & \\
\hline $\begin{array}{l}\text { Pointing to virtual } \\
\text { content }\end{array}$ & -0.260 & 0.085 & -.409 & .004 \\
\hline $\begin{array}{l}\text { Verbal answer in a } \\
\text { dialogue with virtual } \\
\text { content }\end{array}$ & -1.182 & 0.447 & -.351 & .012 \\
\hline
\end{tabular}

\subsection{The influence of perceived virtual interactivity on social presence}

To test whether interactivity within the game influences the sensation of presence (H1) we first conducted a correlation analysis between virtual interaction and social presence of virtual characters. We found a positive correlation between the two aspects $(\mathrm{r}=.379, \mathrm{p}=.011, \mathrm{~N}=44)$. A following regression analysis showed according to $\mathrm{H} 1$ that virtual interaction significantly predicted social presence of virtual characters, $b=.379, t(42)=2.65, p<.011$. Virtual interaction also explained a significant proportion of variance for social presence of virtual characters, $R^{2}=.14$, $F(1,44)=7.046, p<.011$.

\subsection{The influence of socio-demographics and collaborative game play on presence}

The socio-demographic data that was collected was also tested in terms of influencing the results (RQ2). ANOVAs were calculated to test a difference between men and women, mixed- or same-sex pairs and if the two players knew each other, were a couple, or did not know each other at all. No significant results were found.

With regard to the question whether the social presence of the team player distracts participants from the virtual content of the game resulting in less social presence of virtual characters we did not find a correlation indicating that the two social presence concepts co-existed and did not influence each other. 


\section{Discussion}

One objective of this study was to identify relationships between objective and subjective measures of presence within a location-aware augmented reality game. In our study we compared the results from the questionnaire with the codings from the video recordings. We identified links between two behavioral measures and subjective data that occurred in both the correlations and the regression models: "pointing to virtual content" and to some degree also "verbal answer in a dialogue with virtual content". These results were found regardless of the player's socio demographic data. The role of these two behavioral elements will be discussed in the context of the calculations made. The items that were not correlated with any presence aspects were "laughing as a reaction to virtual content", "commenting the virtual content", "smiling about virtual content", "making a sound as a reaction to virtual content", and "turning towards virtual figure". This might be true due to the fact that these items contain behavior that is shown more subconscious than answering or pointing. For example smiling and laughing are often activated automatically and may therefore occur in both strong and weak states of presence.

The first result was a combination of pointing and both the feeling of being in the time of the game and the ability to interact with the virtual person. As Slater, Usoh, and Chrysanthou [27] have already mentioned, pointing to something can be interpreted as an indicator for presence. The experimental setting of this study, however, differed strongly from the setting of Slater et al.'s study. In the TimeWarp game all interactions with virtual objects can solely be executed through the UMPC interface. The negative correlation between pointing and the presence measured suggests that in the case of this game pointing is a result of a distraction (e.g. pointing to virtual or real objects) of the game's interaction mode. Since both the feeling of being in the time and being able to interact with virtual content are not directly semantically related to pointing, pointing might be in general a good indicator for presence. Especially the case of temporal presence shows that pointing has an influence on the feeling of presence that is not caused by an obvious connection.

The second result was the negative correlation between the feeling of being able to interact with a virtual character and being able to "speak with them". It should be noted again that all dialogues were controlled by selecting one of several offered multiple-choice options, there was no voice recognition or any other incentive for the players to actually directly talk to the virtual characters. Therefore, a stronger sense of the ability to interact with the virtual content via the interface made the participants use less verbal language. A possible explanation for this may be that if the participants are deeper in the game's own interaction mode (via the UMPC interface) and respect the rules of the interaction more seriously, these participants have a strong sense of presence.

The regression analysis revealed a predictive characteristic of the same behavioral items for the presence aspect virtual interaction and temporal presence. Both concepts could be predicted by the pointing behavior of the participants and the feeling of the possibility to interact with virtual content was additionally predicted negatively by verbal answers in a dialogue with the virtual content. This as well suggests a more general influence on the feeling of 
presence for both talking and especially pointing during a game which can be distracting and not efficiently using the interaction system which solely relies on using the given interface.

These findings differ strongly from Slater et al. [27] who showed that pointing was an indicator for the experience of presence. We assume that the explanatory power of behavioral measures depends greatly on the interaction mode during the (game) experience. While in TimeWarp pointing was not an effective interaction behavior, the setting in Slater et al. required pointing to real and virtual objects. We assume that pointing in general is a reliable behavior to measure the sensation of presence, however, the setting and the associated interaction requirements have to be taken into account. Same counts for the occurrence of verbal interaction. Future research should foster our insights in how these behaviors are linked to the experience of presence and investigate more systematically different interaction modes.

With regard to the influence of interactivity on presence, we were able to show that regardless of an actual manipulation of interactivity, the subjective perception of interaction possibilities with virtual elements of the game influenced the perceived social presence of virtual characters. This suggests that players evaluate the same interaction possibilities provided in our game very differently - a finding that should be taken into account in further investigations on the influence of interactivity on presence.

Contrary to our hypothesis we found that a high social presence of the team partner during game play did not distract participants from the virtual content and did not diminish the social presence towards virtual characters. Moreover, we investigated the role of socio-demographic variables and of the relationships status of the players. We found that the participants' gender, the fact whether they played in same-sex pairs or mixed-sex pairs as well as their relationship status did not influence the experience of presence, although prior research suggests that game play with friends leads to higher enjoyment and arousal [40].

However, the results of the study are subject to some limitations. Firstly, the sample size was limited due to reasons of expense (each run took around two hours in total). A bigger sample could strengthen the results. Also, there was no examination of age differences since age was not systematically tested. The fact that the game was played as a two player game is methodologically not as strong, but was necessary due to reasons of safety and collaboration. However, it was found in this study that the quota how much the players played in each mode had no impact on the results. Nevertheless, future research could conduct a similar investigation that uses a single-player game. However, an assumption for a single-player setting could be that the players use less speech and pointing gestures in general, because no real person stimulates a conversation or nonverbal interaction.

Another limitation that has to be acknowledged concerns the video analysis. Here as well due to reasons of expense, the analysis was limited to only one scene, although the self-report of presence that was measured with the questionnaire displayed the feeling of presence at the end of the game when all scenes had been played. It may be possible that other scenes in the game influenced the sensation of presence more than the wedding scene. Therefore future evaluation approaches could focus on measuring presence at various points during the experience and not 
simply at the end. Also it would be interesting to use different types of complete presence scales and to compare the results. Additionally, future research could change the design of such a study into an experimental setting, to proof if the correlations found are still consistent after manipulation.

Because the study as a whole lasted more than two hours on average we used a very shortened version of the traditional presence questionnaires (Networked Minds Questionnaire [24]; MEC Questionnaire [43]) resulting in quite low reliability for higher cognitive involvement. We suggest using full subscales for better reliability of the selfreported measures in further studies.

The paper presented a study about behavioral and subjective measures of presence. However, the behavioral measures that are relevant for this game are context specific and are not necessarily relevant for every game. It is noteworthy that from the variety of behavioral measures which were both related to the virtual content and to the collaboration partner only few measures were able to predict merely a few presence self-report measures.

Additionally, the genre of the chosen game needs to be taken into account as well. For example in an augmented reality game the player has a systematically different experience than in a virtual environment. The sensation of presence in an AR game includes both the real and virtual space. Therefore, the findings of this paper might only be true for AR games and differ in virtual environments.

\section{Conclusions}

The study presented focused on the question which behavioral elements accompany the sensation of presence. Results showed in different tests the behaviors "verbal answer in dialogue with virtual content" and "pointing to virtual content" to be connected negatively to the experience of temporal presence and virtual interaction, respectively. While especially pointing has been proven to be connected positively to the sensation of presence before it remains unclear in which settings pointing and verbal interaction is a positive or negative indicator for presence. However, in conclusion it can be said that there are behavioral elements that are connected to a self-reported sense of presence.

\section{Acknowledgements}

TimeWarp was created as part of the EC-funded IPCity project. The authors would like to thank the project members and especially the staff and students at Fraunhofer FIT who developed the game and conducted the user study on which this work is based.

\section{References}

[1] W. Broll, A.-K. Braun, I. Herbst, R. McCall, R. TimeWarp: Interactive Time Travel with a Mobile Mixed Reality Game. In Proc. of ACM MobileHCI 2008, the 10th International Conference on Human-Computer Interaction with Mobile Devices and Services. 2008. 
[2] M. Lombard, T. Ditton. At the heart of it all: The concept of presence. In Journal of Computer Mediated Communication 3(2/97).

[3] B. G. Witmer, M.J. Singer. Measuring presence in virtual environments: A presence questionnaire. Presence: Teleoperators and Virtual Environments, 7/98, 225-240.

[4] C. Heeter. Being There: The Subjective Experience of Presence. In Presence: Teleoperators and Virtual Environments, 1(2/92), 262-271.

[5] W. IJsselsteijn, G. Riva. Being there: the experience of presence in mediated environments. In: Riva, G.; Davide, F.; IJsselsteijn, W.A. (Eds.): Being There: Concepts, effects and measurement of user presence in synthetic environments. Amsterdam: Ios Press. 2003.

[6] K. M. Lee. Presence, Explicated. In Communication Theory, 14(1/04), 27-50.

[7] C. Klimmt, T. Hartmann. Effectance, self-efficacy, and the motivation to play video games. In P. Vorderer \& J. Bryant (Eds.), Playing video games: Motives, responses, and consequences. Mahwah: Lawrence Erlbaum Associates. 2006.

[8] R. Tamborini, M. Eastin, P. Skalski, K. Lachlan, T. Fediuk, R. Brady. Violent virtual video games. In Journal of Broadcasting and Electronic Media, 48(3/04), 335-357.

[9] L. Reinecke, J. Klatt, N. C. Krämer. Entertaining Media Use and the Satisfaction of Recovery Needs: Recovery Outcomes Associated With the Use of Interactive and Noninteractive Entertaining Media. In Media Psychology, 14(2/11), 192-215.

[10] P. Vorderer, S. Knobloch, H. Schramm. Does Entertainment Suffer From Interactivity? The Impact of Watching an Interactive TV Movie on Viewers' Experience of Entertainment. In Media Psychology, 3(4/01), 343-363.

[11] S. Calvert, S.-L. Tan. Impact of virtual reality on young adults' physiological arousal and aggressive thoughts: Interaction versus observation. In Journal of applied developmental psychology, 15(1/94), 125-139.

[12] R. B. Welch, T. T. Blackmon, A. Liu, B. A. Mellers, L. W. Stark. The effects of pictorial realism, delay of visual feedback, and observer interactivity and the subjective sense of presence. In Presence-Teleoperators and Virtual Environments, 5/96, 263-273.

[13] R. Tamborini, M. Eastin, K. Lachlan, P. Skalski, T. Fediuk, R. Brady. Hostile Thoughts, Presence and Violent Video Games. Paper presented at the 51st annual convention of the International Communication Association, Washington, D.C. 2001.

[14] L. Hoffmann, J. Klatt, A. Lam-chi, N. Haferkamp, N. C. Krämer. Looking through the eyes of an avatar - how point of view influences the perception of virtual groups. Talk at the International Communication Association, Singapore. 2010. 
[15] P. Larsson, D. Västfjäll, P. Olsson, M. Kleiner. When what you see is what you hear: Auditory-visual integration and presence in virtual environments. In Proceedings of the 10th Annual International Workshop Presence, Barcelona, Spain. 2007.

[16] M. Usoh, E. Catena, S. Arman, M. Slater. Using presence questionnaires in reality. In Presence-Teleoperators and Virtual Environments 9/00, 497-503.

[17] M. Slater, M. Usoh. Representation Systems, Perceptual Position and Presence in Virtual Environments. In Presence-Teleoperators and Virtual Environments 2/94, 221-234.

[18] J. Blascovich, J. Loomis, A. Beall, K. Swinth, C. Hoyt, J. Bailenson. Immersive virtual environment technology as a research tool for social psychology. In Psychological Inquiry, 13(2/02), 103-124.

[19] F. Biocca, C. Harms, J. L. Gregg. The networked minds measure of social presence: Pilot test of the factor structure and concurrent validity. Proceedings of the Fourth Annual International Presence Workshop, Philadelphia, PA. 2001.

[20] G. Riva, F. Davide, W. A. IJsselsteijn. Being There: Concepts, effects and measurement of user presence in synthetic environments. Amsterdam: Ios Press. 2003.

[21] B. Wissmath, D. Weibel, F.W. Mast. Measuring presence with verbal vs. pictorial scales: A comparison between online and ex post ratings. In Virtual Reality, 14/10, 43-53.

[22] W. A. IJsselsteijn, H. de Ridder, J. Freeman, S.E. Avons. Presence: Concept, determinants and measurement. In Proceedings of the SPIE 3959, 520-529. 2000.

[23] J. Freeman, S. E. Avons, D. E. Pearson, W. A. IJsselsteijn. Effects of sensory information and prior experience on direct subjective ratings of presence. In Presence: Teleoperators and Virtual Environments, 8(1/99), 1-13.

[24] J. N. Bailenson, J. Blascovich, A. C. Beall, J. M. Loomis. Equilibrium revisited: Mutual gaze and personal space in virtual environments. In Presence, 10/01, 583-598.

[25] J. D. Prothero, D. E. Parker, T. A. Furness III, M. J. Wells. Towards a robust, quantitative measure for presence. In Proceedings of the Conference on Experimental Analysis and Measurement of Situation Awareness, 359-366. 1995.

[26] M. Lombard, T. B. Ditton, D. Crane, B. Davis G. Gil-Egui, K. Horvath, J. Rossman, S. Park. Measuring presence: A literature-based approach to the development of a standardized paper-and-pencil instrument. Presented at the Third International Workshop on Presence, Delft, the Netherlands. 2000.

[27] M. Slater, M. Usoh, Y. Chrysanthou. The Influence of Shadows on Presence in Immersive Virtual Environments. In Selected paper of the eurographics workshops on virtual environments '95, 8-21, Barcelona, Spain: Springer. 1995. 
[28] J. Freeman, S. E. Avons, R. Meddis, D. E. Pearson, W. A. IJsselsteijn. Using behavioural realism to estimate presence: A study of the utility of postural responses to motion-stimuli. In Presence: Teleoperators and Virtual Environments, 9 (2/00), 149-165.

[29] M. Slater, C. Guger, G. Edlinger, R. Leeb, G, Pfurtscheller, A Antley, M. Garau, A. Brogni, D. Friedman. Analysis of physiological responses to a social situation in an immersive virtual environment. In Presenceteleoperators and virtual environments, 15 (5/06), 553 - 569.

[30] J. W. Berger, D. S. Shin. Computer-vision-enabled Ophthalmic Augmented Reality: A PC-based Prototype. In Behringer, R., Klinker, G., Mizell, D.W. (Hrsg.). Augmented Reality. Placing artificial objects in real scenes. Canada: Library of Congress cataloging-in-Publication Data. 1990.

[31] R. Azuma. A Survey of Augmented Reality." Presence: Teleoperators and Virtual Environments 6 (4/97), 355385.

[32] D. Reiners, D. Stricker, G. Klinker, S. Müller. Augmented reality for Construction Tasks: Doorlock Assembly. In Behringer, R., Klinker, G., Mizell, D.W. (Hrsg.). Augmented Reality. Placing artificial objects in real scenes. Canada: Library of Congress cataloging-in-Publication Data. 1999.

[33] W. Höhl. Interactive Environments with Open-Source Software. 3D walkthroughs and Augmented reality for Architects with Blender 2.43, Dart 3.0 and ARToolKit 2.72. Wien: Springer. 2009.

[34] G. Simon, V. Lepetit, M.-O. Berger. Computer Vision Methods for Registration: Mixing 3D Knowledge and 2D Correspondences for Accurate Image Composition. In Behringer, R., Klinker, G., Mizell, D.W. (Eds.). Augmented Reality. Placing artificial objects in real scenes. Canada: Library of Congress cataloging-inPublication Data. 1999.

[35] M. Rosenthal, A. State, J. Lee, G. Hirota, J. Ackerman, K. Keller, E. D. Pisano, M. Jiroutek, K. Muller, H. Fuchs. Augmented Reality Guidance for Needle Biopsies: A Randomized, Controlled Trial in Phantoms. In Proc. Medical Image Computing and Computer-Assisted Intervention 2001, 240-248. 2001.

[36] W. Piekarski, B. Thomas. ARQuake: The Outdoor Augmented Reality Gaming System. Communications of the ACM, 45/02, 36-38.

[37] 3DS Nintendo Life (2011). Hands On: Nintendo 3DS Augmented Reality Games. URL: http://3ds.nintendolife.com/news/2011/03/hands_on_nintendo_3ds_augmented_reality_games

[38] A. Morrison, D.Stuedahl, C. Mörtberg, I. Wagner, G. Liestøl, T. Bratteteig. Analytical Perspectives. In Wagner, I., Bratteteig, T., \& Stuedahl, D. (Eds.), Exploring Digital Design: Multi-disciplinary design practices. Springer Verlag London. 2010.

[39] B. Nardi, J. Harris. Strangers and friends: collaborative play in world of warcraft. In CSCW '06: Proceedings of the 2006 20th anniversary conference on Computer supported cooperative work (pp. 149-158). ACM Press. 2006. 
[40] N. Ravaja, T. Saari, M. Turpeinen, J. Laarni, M. Salminen, M. Kivikangas. Spatial presence and emotions during video game playing: Does it matter with whom you play? Presence, Vol. 15, No. 4, August 2006, 381-392, 2006.

[41] K. Satoh, T. Ohshima, H. Yamamoto, H. Tamura. Case Studies of See-Through Augmentation in Mixed Reality Project. In Behringer, R., Klinker, G., Mizell, D.W. (Hrsg.). Augmented Reality. Placing artificial objects in real scenes. Canada: Library of Congress cataloging-in-Publication Data, 3--18, 1999.

[42] T. Starner, B. Leibe, B. Singletary, and J. Pair. "MIND-WARPING: Towards Creating a Compelling Collaborative Augmented Reality Game." In Proc. of ACM Intelligent User Interfaces, New Orleans, LA, January 2000.

[43] P. Vorderer, W. Wirth, F. R. Gouveia, F. Biocca, T. Saari, F. Jäncke, S. Böcking, H. Schramm, A. Gysberg, T. Hartmann, C. Klimmt, J. Laarni, N. Ravaja, A. Sacau, T. Baumgartner, P. Jäncke. MEC Spatial Presence Questionnaire (MEC-SPQ): Short documentation and Instructions for Application. In Report to the European Community, Project Presence: MEC. 2004. 